\title{
Effect of TH on osteoclastogenesis and differentiation
}

\section{Hitoshi Amano, Masakazu Inubushi, Futoshi Iwaki, Kiyoshi Ohura}

Pharmacology, Faculty of Dentisty, Osaka Dental University, Japan

A helioxanthin-derivative, 4-(4-methoxyphenyl)pyrido [4',3':4,5] thieno [2,3-b] pyridine-2-carboxamide (TH), induced osteogenic differentiation in MC3T3-E1 cell. The combination of TH and bone morphogenetic protein(BMP) 2 induced the mRNA expression of osteoblast marker genes and calcification in primary fibroblasts. We examined whether TH could inhibit osteoclast differentiation in vitro. Using the hematopoietic stem cells from the femour and the tibias of ddY mice, $\mathrm{TH}$ was added to the culture in the experimental group, and the number of osteoclast was measured with rhodamine phalloidin staining and TRAP staining. In osteo assay, bone resorption area was compared by the von Kossa staining. TH has been demonstrated that transient increase of NO is deeply involved in osteoclast differentiation. The number of TRAP-positive multinucleated cells and F-actin rings were both decreased depending on TH concentration. The bone resorption area was reduced in a concentration-dependent manner of TH. In order to verify the pharmacological action, inhibition recovery experiment was conducted by adding soluble guanylyl cyclase inhibitor (ODQ) in osteoclast formation process. Inhibitory effect of TH on osteoclastogenesis was blocked by addition of ODQ. These results suggest that both action of TH induced NO inhibit osteoclast formation and differentiation. 